\title{
Effects of outcome and trial frequency on the inverse base-rate effect
}

\author{
Hilary J. Don ${ }^{1}$ - Evan J. Livesey ${ }^{1}$
}

Published online: 4 November 2016

(C) Psychonomic Society, Inc. 2016

\begin{abstract}
The inverse base-rate effect is a bias in contingency learning in which participants tend to predict a rare outcome for a conflicting set of perfectly predictive cues. Although the effect is often explained by attention biases during learning, inferential strategies at test may also contribute substantially to the effect. In three experiments, we manipulated the frequencies of outcomes and trial types to determine the critical conditions for the effect, thereby providing novel tests of the reasoning processes that could contribute to it. The rare bias was substantially reduced when the outcomes were experienced at equal rates in the presence of predictive-cue frequency differences (Exp. 2), and when the predictive cues were experienced at equal rates in the presence of outcome frequency differences (Exp. 3). We also found a consistent commonoutcome bias for novel cue compounds. The results indicate the importance of both cue and outcome frequencies to the inverse base-rate effect, and reveal a combination of necessary conditions that are not well captured by appealing to inferential strategies at test. Although both attention-based and inferential theories explain some aspects of these data, no existing theory fully accounts for these effects of relative novelty.
\end{abstract}

Keywords Inverse base-rate effect - Contingency learning $\cdot$ Inferential reasoning $\cdot$ Cue competition

Electronic supplementary material The online version of this article (doi:10.3758/s13421-016-0667-y) contains supplementary material, which is available to authorized users.

Hilary J. Don

hdon7006@uni.sydney.edu.au

1 School of Psychology, University of Sydney, Sydney, NSW 2006, Australia
The inverse base-rate effect is a robust and seemingly nonrational bias in human contingency learning, first reported by Medin and Edelson (1988). In their task, participants assumed the role of a doctor diagnosing fictitious diseases on the basis of symptom pairs. Participants learned which symptoms indicated which diseases through trial and error with corrective feedback. All patients with symptom A and symptom B had disease O1, whereas all patients with symptom A and symptom $\mathrm{C}$ had disease O2. Disease O1 occurred three times as often as O2. Symptom A is therefore an imperfect predictor, because it is paired with both diseases. Symptom B is a perfect predictor of the common disease, $\mathrm{O} 1$, and symptom $\mathrm{C}$ is a perfect predictor of the rare disease, O2. After learning these contingencies, participants completed a transfer phase including several new symptom combinations. When presented with symptom A, participants diagnosed the common disease. Although symptom A predicted both diseases, this response is consistent with the underlying base rates of the two diseases. Participants also diagnosed the common disease when presented with combined symptoms, ABC. However, when presented with conflicting symptoms, BC, participants diagnosed the rare disease, a response that goes against the underlying base rates of the associated diseases. The inverse base-rate effect refers to this bias in choosing the rare outcome for conflicting cues, and it has been shown in a variety of learning tasks (Johansen, Fouquet, \& Shanks, 2010; Kalish, 2001; Lamberts \& Kent, 2007). The basic design of the task is shown in Table 1, in which letters represent the individual cues, and $\mathrm{O} 1, \mathrm{O} 2$, and so forth represent the individual outcomes, such that $\mathrm{AB}-\mathrm{O} 1$ occurs three times as often as $\mathrm{AC}-\mathrm{O} 2{ }^{1}$

\footnotetext{
${ }^{1}$ Note that where there are multiple instantiations of the design, the letters A$\mathrm{C}$ will refer to all cues of the same type.
} 
Table 1 Basic inverse base-rate task design

\begin{tabular}{llll}
\hline \multirow{3}{*}{ Base Rate } & Training Phase Trials & Transfer Phase & \\
\cline { 3 - 4 } & & Type & Trials \\
\hline 3 & $\mathrm{~A} B-\mathrm{O} 1$ & Imperfect Predictors & $\mathrm{A} ?$ \\
1 & $\mathrm{AC}-\mathrm{O} 2$ & Conflicting transfer & $B \mathbf{C} ?$ \\
& & Combined transfer & $\mathrm{A} B \mathbf{C}$ ? \\
\hline
\end{tabular}

A-C represent different symptom cues, O1-O2 represent different disease outcomes. $\mathrm{AB}-\mathrm{O} 1$, for example, indicates that symptom $\mathrm{A}$ and symptom $\mathrm{B}$ predicted disease O1. Cues in italics indicate perfect predictors of common outcomes, and cues in bold indicate perfect predictors of rare outcomes. "Base rate" refers to the relative number of presentations of each trial type during training, such that $\mathrm{AB}-\mathrm{O} 1$ occurs three times as often as AC-O2. ? indicates trials on which participants make a response without feedback.

\section{Explanations for the inverse base-rate effect}

The effect is prominently explained as a result of cue competition during learning, due to error-driven selective attention processes. Currently, the EXIT model provides the best account of this process (Kruschke, 1996, 2001b). According to EXIT, the common cue-outcome contingencies are learned more quickly than the rare contingencies, such that both $\mathrm{A}$ and $\mathrm{B}$ become associated with the common outcome, $\mathrm{O} 1$. To reduce subsequent error on $\mathrm{AC}-\mathrm{O} 2$ trials, attention is directed away from the ambiguous cue $\mathrm{A}$, and toward the perfect predictor, $\mathrm{C}$. This results in a stronger association between $\mathrm{C}-\mathrm{O} 2$ than between $\mathrm{B}-\mathrm{O} 1$, such that $\mathrm{C}$ tends to control responding on $\mathrm{BC}$ trials. Although the inverse baserate effect has been an important phenomenon for the development and subsequent defense of attention-based associative-learning theories (e.g., Le Pelley, Mitchell, Beesley, George, \& Wills, 2016), some findings are clearly less consistent with this approach, leading some theorists to regard the effect as the consequence of a reasoned strategy on test.

\section{Inferential explanations}

Several researchers have suggested that higher-order reasoning contributes to the effect (Johansen, Fouquet, \& Shanks, 2007; Kruschke, 2003; Kruschke, Kappenman, \& Hetrick, 2005; Winman, Wennerholm, \& Juslin, 2003). Primary evidence for this view has come from Winman, Wennerholm, Juslin, and Shanks (2005). First, they compared the effects between adults and children, with the assumption that children would be more likely to rely on simple associative processes than on high-level reasoning. Whereas adults showed a clear bias, children showed no bias in outcome choices on conflicting trials. This difference in the response patterns between groups was not attributable to differences in learning efficiency, indicating that the result was not simply due to differences in learned associations or selective attention. Second, the researchers classified participants as either rule-based or featurebased, according to their generalization performance in a separate patterning task, in which rule transfer is associated with higher working memory capacity (Wills, Barrasin, \& McLaren, 2011), greater cognitive reflection, and more strategic "model-based" choices in reinforcement learning (Don, Goldwater, Otto, \& Livesey, 2016). Only those participants who were able to extract and apply an abstract rule in the patterning task exhibited an inverse base-rate effect. Furthermore, Johansen et al. (2007) demonstrated an effect when the cue-outcome contingencies were provided simultaneously in a written, listed format, suggesting that trial-by-trial learning is not necessary. Such results are not readily explained by attention theories and, taken together, suggest that inferential processes play a role in the effect.

\section{Limitations of existing inferential accounts}

Currently, the sole inferential account of the inverse base-rate effect is eliminative inference, formalized in the elimination model (ELMO; Juslin, Wennerholm, \& Winman, 2001). Informally, eliminative inference states that, given an ambiguous cue combination that deviates from the learned contingency rules, the well-known common outcome is eliminated, and the rare outcome is thus more likely to be chosen. Initial support for eliminative inference came from novel-cue effects (Juslin et al., 2001). According to eliminative inference, a novel cue should elicit elimination, due to its dissimilarity to the rules learned in training. Since the rules that are active in memory are more likely to involve a common outcome, the participant is more likely to guess a rare outcome. In accordance with this view, Juslin et al. found greater rare-outcome responses for a single novel cue during transfer, similar to the results from conflicting transfer trials. This result has since been replicated in a simple decision-making version of the task (Johansen et al., 2007).

Eliminative inference is often interpreted as a novelty effect (Johansen et al., 2007; Juslin et al., 2001; Kruschke, 2001a); that is, participants assume that a novel or ambiguous cue combination requires a novel response. The rare outcome is more "novel" than the common outcome, so this is chosen on conflicting trials. Although in many circumstances it is practical to consider this novelty-matching inference and elimination as being equivalent, it is worth noting that these two inferences are distinct in form and function. Perhaps the most salient difference is that rather than eliminating an unlikely response and guessing among the remaining options, novelty matching involves selecting the most novel outcome given a novel cue combination.

ELMO fails to account for several characteristics of the effect, including the necessity of a shared cue during training 
and differences in the strength of responding to imperfect and combined transfer items (Kruschke, 2001a; Wills, Lavric, Hemmings, \& Surrey, 2014). As such, it is no longer considered a plausible model of the effect.

\section{Problems with discounting inferential contributions}

The limitations of one particular formalization of eliminative inference certainly do not rule out the involvement of inference entirely. Nor does it prohibit the involvement of outcome elimination or novelty matching in some general form, albeit in a way that may be different from that specified by ELMO. Indeed, the demonstrated rare biases for novel cues may rely on such explanations (Johansen et al., 2007; Juslin et al., 2001). Winman et al.'s (2005) results do not specifically support ELMO, but rather suggest the involvement of high-level processes of some kind, and it seems likely that participants reason about their decisions in an experiment, particularly when faced with ambiguous information. Moreover, inferential accounts remain important potential explanations of other cue-competition effects, such as blocking (Mitchell, De Houwer, \& Lovibond, 2009), and there is evidence that both associative learning and inference contribute to the strength of the learned-predictiveness effect (Don \& Livesey, 2015). Thus, it appears premature to reject the possibility that some form of inference could result in the inverse base-rate effect. This conclusion is consistent with the dual-process view, which assumes that associations and inferences are both important for human learning (McLaren et al., 2014). However, in order to understand how these processes could interact, we must first characterize the kinds of inferential reasoning that may plausibly be involved in the effect.

\section{The present study}

In the present study, we manipulated experimental parameters in the Medin and Edelson (1988) task to fulfill two aims: first, to extend our understanding of the critical boundary conditions of the effect, and second, to provide a test of the kinds of inferences that could plausibly lead to rare outcome choices on conflicting trials. These manipulations included the relative novelty of transfer trials (Exps. 1-3), the relative frequency of outcomes across training (Exp. 2), and the relative frequencies of predictive cues across training (Exp. 3). The importance of these conditions will be explained in further detail in the following experiments. These experiments were not intended to differentiate directly between associative and inferential accounts, but rather to test the key assumptions on which inferential strategies could be based. As we will discuss, the experiments tested the sensitivity of the effect to the relative novelty of cues and outcomes, which associative or inferential explanations could potentially speak to in different ways.

\section{Experiment 1}

Experiment 1 was a preliminary experiment with two important purposes. First, it introduced a novel-transfer test that was used in the following set of experiments. Evidence for rare responses to novel cues indicate that participants might still engage in outcome elimination or novelty matching of some form, wherein they favor novel outcomes on ambiguous trials. This provides a clear starting point to characterize their potential reasoning processes. Any form of elimination or novelty matching would assume that, all else being equal, the similarity between the trained and test trials should be related to the probability of choosing the rare over the common outcome; when cue similarity is lower, the probability of choosing the rare outcome is higher. Rare-outcome biases have only been demonstrated for a single novel cue in the absence of a rare bias for conflicting cues (Johansen et al., 2007; Juslin et al., 2001). It is plausible that a completely novel cue could elicit a different response strategy to that used on conflicting transfer trials, which are novel combinations of previously experienced cues. Therefore, the novel transfer in the present study consisted of an imperfect predictor paired with a novel cue (e.g., AW).

An account based on simple generalization of learned associations predicts that participants would respond to AW in much the same way as they would respond to A. However, if participants eliminate a familiar outcome or select the most novel outcome according to the similarity of the transfer trials, then responding should be different for $\mathrm{A}$ and $\mathrm{AW}$. Test trial $\mathrm{A}$ differs from trials $\mathrm{AB}$ and $\mathrm{AC}$ in the omission of just one predictive cue, and thus are similar enough to the learned conditions to make choices consistent with the base rates. By comparison, $\mathrm{BC}$ differs from both $\mathrm{AB}$ and $\mathrm{AC}$ in the omission of the imperfect predictor $\mathrm{A}$ and the addition of a perfect predictor, so it is sufficiently dissimilar to warrant a rare choice. It is reasonable to assume that the addition or omission of a predictive cue is more salient than the addition or omission of a nonpredictive cue, because it is well known that more attention is paid to predictive cues. However, there is also good reason to believe that novel cues are relatively salient. Thus, AW differs from each of the trained compounds AB/AC by the (salient) omission of a predictive cue and the (presumably salient) addition of a novel cue, and is likely to be even less similar than $\mathrm{BC}$ to the trained compounds. AW should therefore result in rare outcome choice at least as frequently as BC.

The second aim of Experiment 1 was to test whether the effect was weakened when each outcome was predicted by 
multiple cues. In typical inverse base-rate experiments, every outcome is paired exclusively with only one cue compound (e.g., $\mathrm{O} 1$ only occurs in $\mathrm{AB}-\mathrm{O} 1$ ). To the best of our knowledge, only one previous study has demonstrated the inverse base-rate effect in a task in which multiple cue compounds were paired with the same outcome (Wills et al., 2014). However, these conditions have never been directly compared. Subsequent experiments in the present study require the same outcome to be paired with multiple cue compounds; therefore, in Experiment 1 we aimed to confirm that this would not reduce the strength of the effect. In a "sharedoutcome" condition, four outcomes were each paired with two separate and nonoverlapping cue compounds. This group was compared to two other groups presented with exclusive outcome pairings, matched to the shared-outcome group in either the number of cue compounds ("cue matched") or the number of outcomes ("outcome matched").

\section{Method}

Participants Seventy-eight undergraduates participated in return for partial course credit (62 female, mean age $=$ 20.55 years, $S D=3.88)$. Participants were randomly allocated to three groups $(n=26)$.

Apparatus and stimuli The experiment was programmed using Psychophysics Toolbox for MATLAB (Brainard, 1997; Pelli, 1997) and was presented using Apple Mac Mini computers attached to 17 -in. displays. The experimental stimuli included 16 symptom names: sneezing, dizziness, headache, fever, rash, nausea, earache, coughing, hair loss, fatigue, twitching, blurred vision, wheezing, swelling, back pain, and fainting. The disease outcomes included buragamo, coralgia, midosis, terrigitis, namitis, burlosis, althrax, and gouphosis. For each participant, the symptoms and diseases were randomly allocated to Cues $\mathrm{A}-\mathrm{L}$ and $\mathrm{W}-\mathrm{Z}$ and Outcomes $1-8$, respectively.

Design The trial contingencies are presented in Table 2. In the shared-outcome group, the basic design was repeated four times, with different symptom cues for each instantiation. The common cue compounds (e.g., AB) were presented three times as often as the rare cue compounds (e.g., AC). Each cue compound was paired with one of two common outcomes (O1 or $\mathrm{O} 3)$ or one of two rare outcomes $(\mathrm{O} 2$ or $\mathrm{O} 4)$, such that two different cue compounds predicted the same outcome. The cue-matched condition had identical cue compounds, but each was paired with one of eight unique outcomes (four common, four rare). The outcome-matched group had the same number of outcomes as the shared-outcome group, with only two instantiations of the design. In both the cue- matched and outcome-matched conditions, only one cue compound predicted each outcome.

Procedure Participants assumed the role of a doctor whose task was to learn to make medical diagnoses from experiences with patients exhibiting various symptoms (Fig. 1). On each training trial, two symptom cues appeared in the upper half of the screen, and participants were asked to make a diagnosis by clicking on one of the disease options presented beneath. In the shared-outcome and outcome-matched groups, four outcome options were presented. In the cue-matched condition, eight outcome options were presented. Once an outcome was selected, corrective feedback was provided. The positions of the cues were counterbalanced within each block, and the positions of the outcomes were randomized for each participant. There were seven blocks of training with a 3:1 base rate; each block contained six presentations of each common trial type and two presentations of each rare trial type.

In the transfer phase, participants were instructed to use the knowledge that they had gained so far to diagnose several new patients without feedback. On each trial, one, two, or three symptoms appeared, and participants made their choice by clicking an outcome. They then rated their confidence that they had made the correct choice, on a scale ranging from not at all confident to very confident. Responding was self-paced, and participants were able to modify their response before moving to the next trial. Each transfer trial was presented once and in random order. The positions of cues were randomized for each trial, and the outcome options were presented in the same positions as in training.

Data analysis Our analysis of the transfer phase focused on outcome choice for the four most critical transfer items, including the conflicting, imperfect, combined, and novel cues (see Table 3 for the outcome choice proportions and mean confidence ratings for all transfer trials). To determine choice bias, we calculated the number of relevant rareoutcome choices as a proportion of all relevant outcome choices, following Medin and Edelson (1988). This measure has the benefit of being comparable to an absolute value of .5 reflecting equal choice of the relevant common and rare outcomes. We will refer to the .5 value as "chance"-level responding, since it reflects no preference for either relevant outcome.

There is little consensus about the most appropriate way to statistically analyze the choice bias in the inverse base-rate effect. Many have adopted the approach of using simple $t$ tests, even though the underlying distributions of choice probability may deviate from normality. We have adopted this approach for consistency with previous research, but have also added Bayes factor (BF) tests that do not rely on the same assumptions as traditional parametric null-hypothesis tests. 
Table 2 Design of Experiment 1

\begin{tabular}{|c|c|c|c|c|c|}
\hline \multirow[t]{2}{*}{ Base Rate } & \multicolumn{3}{|l|}{ Training Phase } & \multicolumn{2}{|l|}{ Transfer Phase } \\
\hline & Shared Outcome & Cue Matched & Outcome Matched & Type & Trial \\
\hline 3 & $\mathrm{~A} B-\mathrm{O} 1$ & $\mathrm{~A} B-\mathrm{O} 1$ & $\mathrm{~A} B-\mathrm{O} 1$ & Imperfect predictors & $\underline{\mathrm{A}}, \underline{\mathrm{D}}, \mathrm{G}, \mathrm{J}$ \\
\hline 1 & $\mathrm{AC}-\mathrm{O} 2$ & $\mathrm{AC}-\mathrm{O} 2$ & $\mathrm{AC}-\mathrm{O} 2$ & Conflicting transfer & $\underline{B \mathbf{C}}, \underline{E \mathbf{F}}, H \mathbf{I}, K \mathbf{L}$ \\
\hline 3 & $\mathrm{D} E-\mathrm{O} 1$ & $\mathrm{D} E-\mathrm{O} 3$ & $\mathrm{D} E-\mathrm{O} 3$ & Combined transfer & $\underline{\mathrm{A} B \mathbf{C}}, \underline{\mathrm{D} E \mathbf{F}}, \mathrm{G} H \mathbf{I}, \mathrm{J} K \mathbf{L}$ \\
\hline 1 & $\mathrm{DF}-\mathrm{O} 2$ & $\mathrm{DF}-\mathrm{O} 4$ & $\mathrm{DF}-\mathrm{O} 4$ & Novel transfer & $\underline{\mathrm{AW}}, \underline{\mathrm{DX}}, \mathrm{GY}, \mathrm{JZ}$ \\
\hline 3 & $\mathrm{G} H-\mathrm{O} 3$ & $\mathrm{GH}-\mathrm{O} 5$ & & Perfect common & $\underline{B}, \underline{E}, H, K$ \\
\hline 1 & $\mathrm{GI}-\mathrm{O} 4$ & GI-O6 & & Perfect rare & $\underline{\mathbf{C}}, \underline{\mathbf{F}}, \mathbf{I}, \mathbf{L}$ \\
\hline 3 & $\mathrm{~J} K-\mathrm{O} 3$ & $\mathrm{~J} K-\mathrm{O} 7$ & & Trained common & $\underline{\mathrm{A} B}, \underline{\mathrm{D} E}, \mathrm{G} H, \mathrm{~J} K$ \\
\hline 1 & $\mathbf{J}-\mathrm{O} 4$ & $\mathbf{J L}-\mathrm{O} 8$ & & Trained rare & $\overline{\mathrm{AC}}, \overline{\mathrm{DF}}, \mathrm{GI}, \mathbf{J L}$ \\
\hline
\end{tabular}

A-L represent different symptom cues, O1-O8 represent different disease outcomes. Cues in italics indicate perfect predictors of the common outcomes, and cues in bold indicate perfect predictors of the rare outcomes. Participants in the outcome-matched condition received only the first two trials of each transfer type (underlined). "Base rate" refers to the relative number of presentations of each trial type during training, such that $\mathrm{AB}, \mathrm{DE}, \mathrm{GH}$, and JK are common compounds, and $\mathrm{AC}, \mathrm{DF}, \mathrm{GI}$, and JL are rare compounds.

A full description of these BF tests can be found in the supplementary materials. In brief, the BF tests of the inverse baserate effect for individual groups compared the null hypothesis that relevant outcome choices were unbiased (i.e., equally likely to be common or rare) to the alternative hypothesis that the relevant outcome choices would be biased toward the rare over the common outcome. Thus a BF firmly in favor of the alternative would indicate evidence for an effect. BF tests of the group differences tested whether the key experimental

a

Nausea

Fatigue

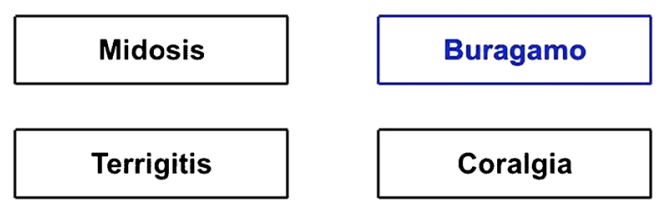

b

\section{Sneezing \\ Back pain}

Which disease is this patient likely to have?

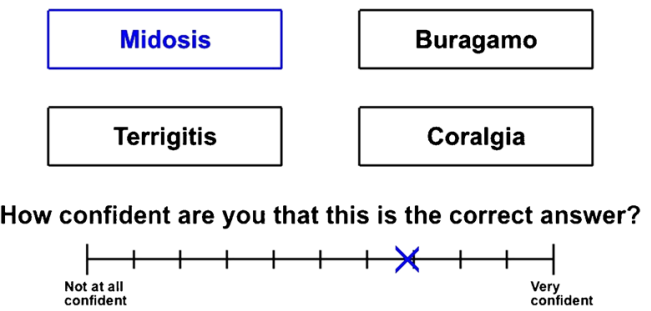

Fig. 1 Example screenshots of (a) the training phase and (b) the transfer phase of the task. On viewing the symptoms (cues) presented for a given trial, participants selected from the available fictitious disease (outcome) choices, followed by either corrective feedback (training only) or a confidence rating (transfer phase only) manipulation weakened the effect. Thus, they compared a null hypothesis that this bias was equal in each group to a directional alternative that less bias was present in the experimental than in the relevant control group.

\section{Results}

To ensure that participants had adequately learned the cue-outcome contingencies, an arbitrary training criterion of $60 \%$ accuracy in the final three blocks of training was set and applied across all experiments. ${ }^{2}$ All of the participants in Experiment 1 met this criterion and remained in the following analyses. The average accuracy across the final three blocks was high $(96 \%$; see the supplementary materials for detailed analyses of the training accuracy and confidence ratings).

Figure 2 shows the average proportions of rare choices for each of the critical transfer trials, and Table 3 shows the choice proportions for all transfer trials. The first result of interest was whether the inverse base-rate effect was reduced in the shared-outcome group relative to the remaining groups. Importantly, rare choice on conflicting trials was significantly greater than chance in all three groups, indicating a significant effect, lowest $t(25)=$ $2.50, p=.019, d=0.49$, lowest $\mathrm{BF}_{10}=4.39$ (cue-matched group). We also found no significant group differences for any of the critical transfer trials, with results ranging from $F(2,77)=0.142, p=.868, \eta^{2}<.01$, for novel trials, to

\footnotetext{
${ }^{2}$ The learning criterion of $60 \%$ is an arbitrary criterion widely used in the literature on other cue-competition effects. We adopted this criterion because there does not appear to be a common learning criterion standard in the inverse base-rate effect. Where some studies have a fairly strict criterion (e.g., Juslin et al., 2001; Winman et al., 2005), others do not apply any learning criterion (e.g., Johansen et al., 2007, 2010; Wills et al., 2014). It is worth noting that applying a stricter criterion (e.g., $85 \%$ correct in the final block) produced the same pattern of results for the critical analyses in each experiment.
} 
Table 3 Outcome choice proportions and mean confidence ratings for all transfer trials in Experiment 1

\begin{tabular}{|c|c|c|c|c|c|c|}
\hline \multirow[b]{2}{*}{ Transfer Trial } & \multirow[b]{2}{*}{ Group } & \multicolumn{4}{|c|}{ Choice Proportion } & \multirow[b]{2}{*}{ Confidence } \\
\hline & & Common & Rare & Other Common & Other Rare & \\
\hline Imperfect & Shared-outcome & .798 & .173 & .029 & 0 & 64.03 \\
\hline \multirow[t]{2}{*}{$\mathrm{A}, \mathrm{D}, \mathrm{G}, \mathrm{J}$} & Cue-matched & .673 & .260 & .048 & .019 & 57.46 \\
\hline & Outcome-matched & .846 & .115 & .019 & .019 & 63.79 \\
\hline Conflicting & Shared-outcome & .231 & .740 & .010 & .019 & 70.81 \\
\hline \multirow[t]{2}{*}{ BC, EF, HI, KL } & Cue-matched & .365 & .596 & .019 & .019 & 60.42 \\
\hline & Outcome-matched & .212 & .769 & .019 & 0 & 70.00 \\
\hline Combined & Shared-outcome & .413 & .577 & .010 & 0 & 70.59 \\
\hline \multirow[t]{2}{*}{ ABC, DEF, GHI, JKL } & Cue-matched & .510 & .471 & 0 & .019 & 62.43 \\
\hline & Outcome-matched & .404 & .596 & 0 & 0 & 68.73 \\
\hline Novel & Shared-outcome & .702 & .221 & .048 & .029 & 49.58 \\
\hline \multirow[t]{2}{*}{ AW, DX, GY, JZ } & Cue-matched & .654 & .260 & .067 & .019 & 49.02 \\
\hline & Outcome-matched & .673 & .231 & .077 & .019 & 58.14 \\
\hline Perfect common & Shared-outcome & .933 & 0 & .067 & 0 & 73.04 \\
\hline \multirow[t]{2}{*}{$\mathrm{B}, \mathrm{E}, \mathrm{H}, \mathrm{K}$} & Cue-matched & .913 & .019 & .038 & .029 & 69.02 \\
\hline & Outcome-matched & .981 & 0 & .019 & 0 & 74.11 \\
\hline Perfect rare & Shared-outcome & .029 & .933 & .019 & .019 & 82.11 \\
\hline \multirow[t]{2}{*}{$\mathrm{C}, \mathrm{F}, \mathrm{I}, \mathrm{L}$} & Cue-matched & 0 & .942 & .010 & .048 & 71.83 \\
\hline & Outcome-matched & .038 & .962 & 0 & 0 & 81.17 \\
\hline Trained common & Shared-outcome & .971 & .010 & .010 & .010 & 93.33 \\
\hline \multirow[t]{2}{*}{$\mathrm{AB}, \mathrm{DE}, \mathrm{GH}, \mathrm{JK}$} & Cue-matched & .981 & .010 & 0 & .010 & 91.11 \\
\hline & Outcome-matched & .981 & 0 & 0 & .019 & 94.54 \\
\hline Trained rare & Shared-outcome & .048 & .923 & .010 & .019 & 87.49 \\
\hline \multirow[t]{2}{*}{ AC, DF, GI, JL } & Cue-matched & .048 & .933 & 0 & .019 & 86.40 \\
\hline & Outcome-matched & .038 & .962 & 0 & 0 & 89.34 \\
\hline
\end{tabular}

$F(2,77)=2.74, p=.071, \eta^{2}=.03$, for conflicting trials. The group difference BFs favored the null when comparing the shared-outcome and cue-matched groups $\left(\mathrm{BF}_{01}=\right.$ 4.65), but were more ambiguous when comparing the shared-outcome and outcome-matched groups $\left(\mathrm{BF}_{01}=\right.$ 2.56). Rare-outcome choice for the imperfect predictor

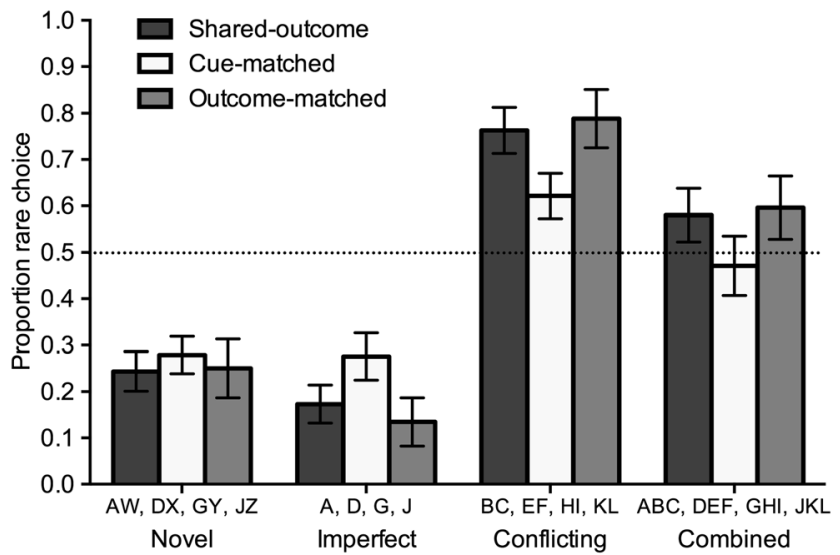

Fig. 2 Mean proportion of relevant rare-outcome choice for the critical transfer items in Experiment 1. Error bars indicate standard errors of the means $(S E M \mathrm{~s})$ was significantly below chance in each group, suggesting a bias toward the relevant common outcome, lowest $t(25)$ $=-4.42, p<.001, d=-0.87$. Choice for the combined transfer did not significantly differ from chance, highest $t(25)=1.41, p=.17, d=0.28$.

The second result of interest was whether participants would show a bias for the rare outcome on novel transfer trials, and whether this would differ from choices on the imperfect and conflicting trials. On novel trials, rare-outcome choice was significantly below chance in all groups, indicating a bias toward the common outcome, lowest $t(25)=-3.93$, $p=.001, d=-0.77$. This bias was compared to the choices on (a) the imperfect trials (A vs. AW tests the effect of adding the novel cue) and (b) the conflicting trials (BC vs. AW tests the effect of similarity on the rare bias) in two separate $(2) \times 3$ mixed measures analyses of variance, including Group as a between-subjects factor. We observed significantly greater rare responses to novel than to imperfect trials, $F(1,75)=4.11$, $p=.046, \eta^{2}=.02$, but rare choice was significantly lower on novel than on conflicting trials, $F(1,75)=89.06, p<.001$, $\eta^{2}=.44$. No significant interactions with group emerged, highest $F(2,75)=1.58, p=.212, \eta^{2}=.02$. 


\section{Discussion}

According to an inference based on outcome elimination or novelty matching, the dissimilarity between the learnedcontingencies and novel-transfer trials should result in a bias toward the rare outcome. Instead, we found a significant bias toward predicting the common outcome. Critically, the proportion of rare outcome choice was significantly lower on novel than on conflicting trials. Although it is possible that outcome choice was controlled entirely by the imperfect predictor in these novel compounds, rare responses were significantly higher on novel than on imperfect trials, which suggests that the presence of a novel cue influenced rare responding to some extent. Although this could indicate some influence of novelty matching, it is also consistent with studies that have shown reduced predictive ratings for trained cues paired with a novel stimulus, compared to ratings for the trained cue alone (Karazinov \& Boakes, 2004; Lotz \& Lachnit, 2009). Nevertheless the results clearly demonstrate that transfer-trial similarity is inadequate as a determinant of common or rare outcome choice. If inference does indeed occur in this context, it is clear that the decision to choose the rare outcome hinges on factors other than just trial similarity.

We found a significant bias toward predicting the rare outcome on conflicting trials, irrespective of whether this outcome was uniquely paired with one cue compound or was a shared outcome for multiple cue compounds. The effect does appear to be slightly weaker in the cue-matched group; however, this difference was not significant, and in any case is difficult to interpret in isolation. In any account that involves an element of guessing, it is possible that guessing the relevant rare outcome is more probable when there are fewer outcome options, which was the case in the shared-outcome and outcome-matched conditions. Yet it is also possible that the cue-matched condition was simply more difficult than the other conditions, given the greater number of outcomes to learn. Most importantly for the experiments to come, the effect in the shared-outcome group was not substantially weaker than in either control group. We thus used this sharedoutcome condition as the control for the following experiments, which teased apart the contributions of general outcome frequency and specific trial frequency to the effect.

\section{Experiment 2}

By design, these tasks involve differences in the frequencies of trial types, such that some are relatively common (AB-O1) and some are relatively rare $(\mathrm{AC}-\mathrm{O} 2)$. Naturally, the base rates of the outcomes themselves differ accordingly. We will refer to this property of the design as the global outcome frequency, to distinguish it from the local frequency of an outcome within a set of overlapping rules such as $\mathrm{AB}-\mathrm{O} 1 /$
AC-O2. A global outcome frequency difference may be a critical condition for the effect. In other words, it may be important that some outcomes are experienced at a greater rate than others during the experiment, so that the outcomes paired with rare cues are clearly more unusual than those paired with common cues at test. This is particularly true of noveltymatching accounts, in which the rare outcome may be chosen specifically for its novelty. In Experiment 2 we therefore tested whether the effect was substantially weakened when there were no differences in global outcome frequency.

The inverse base-rate effect and related highlighting effects have previously been demonstrated with equal cue-outcome base rates. Medin and Bettger (1991) trained participants on a subset of cues with a $3: 1$ base rate, followed by training with a 1:3 base rate, such that each cue-outcome contingency had been experienced equally. In Kruschke's (2009) highlighting design, $\mathrm{AB}-\mathrm{O} 1$ trials were trained prior to $\mathrm{AC}-\mathrm{O} 2$ trials; however, the frequency of each trial type changed across the course of the experiment, so that the base rates were equal by the end of the experiment. In both studies, participants demonstrated a preference for the early rare over the early common outcome on conflicting trials, suggesting the importance of early relative frequencies for the effect.

Although matching cue--outcome contingencies necessarily matches the global frequency of the outcomes, these designs did so by changing the relative frequencies of the trial types across the course of training. This means that for any given block of trials in training, there were still strong global outcome frequency differences. If participants exhibit a primacy effect - that is, stronger learning for events occurring early in training - then one might still expect to see an inverse base-rate effect specifically because judgments of the novelty of the outcomes also reflect this early learning. This possibility means that these designs are relatively uninformative about the role played by outcome and trial frequencies, and they do not rule out any particular explanation of the effect.

In this experiment, we therefore manipulated the overall frequency of the outcomes in a different way: Global outcome frequency was manipulated between groups, while maintaining the relative frequency differences between common and rare trials by using a consistent 3:1 base rate throughout training (see Table 4). Both groups experienced identical presentations of the cue compounds, but the outcome with which each cue compound was paired differed between groups. The standard condition was identical to the shared-outcome condition in Experiment 1. In this condition, $\mathrm{O} 1$ and $\mathrm{O} 3$ were always paired with the common cue compounds, and $\mathrm{O} 2$ and $\mathrm{O} 4$ were always paired with the rare cue compounds. Thus, $\mathrm{O} 1$ and $\mathrm{O} 3$ were experienced three times as often as $\mathrm{O} 2$ and O4. This was compared to a balanced-outcome condition. Here, each outcome was paired with both a common and a rare cue compound, such that all outcomes were experienced with equal frequencies over the course of the experiment. For 
Table 4 Design of Experiment 2

\begin{tabular}{|c|c|c|c|c|}
\hline \multirow[t]{2}{*}{ Base Rate } & \multicolumn{2}{|c|}{ Training Phase } & \multicolumn{2}{|l|}{ Transfer Phase } \\
\hline & Standard & Balanced & Type & Trials \\
\hline 3 & $\mathrm{~A} B-\mathrm{O} 1$ & $\mathrm{~A} B-\mathrm{O} 1$ & Imperfect predictors & $\mathrm{A}, \mathrm{D}, \mathrm{G}, \mathrm{J}$ \\
\hline 1 & $\mathrm{AC}-\mathrm{O} 2$ & $\mathrm{AC}-\mathrm{O} 2$ & Conflicting transfer & $B \mathbf{C}, E \mathbf{F}, H \mathbf{I}, K \mathbf{L}$ \\
\hline 3 & $\mathrm{D} E-\mathrm{O} 1$ & $\mathrm{D} E-\mathrm{O} 2$ & Combined transfer & $\mathrm{A} B \mathbf{C}, \mathrm{D} E \mathbf{F}, \mathrm{G} H \mathbf{I}, \mathrm{J} K \mathbf{L}$ \\
\hline 1 & $\mathrm{DF}-\mathrm{O} 2$ & $\mathrm{DF}-\mathrm{O} 1$ & Novel transfer & AW, DX, GY, JZ \\
\hline 3 & $\mathrm{G} H-\mathrm{O} 3$ & $\mathrm{G} H-\mathrm{O} 3$ & Perfect common & $B, E, H, K$ \\
\hline 1 & GI-O4 & GI-O4 & Perfect rare & $\mathbf{C}, \mathbf{F}, \mathbf{I}, \mathbf{L}$ \\
\hline 3 & $\mathrm{~J} K-\mathrm{O} 3$ & $\mathrm{~J} K-\mathrm{O} 4$ & Trained common & $\mathrm{A} B, \mathrm{D} E, \mathrm{G} H, \mathrm{~J} K$ \\
\hline 1 & $\mathbf{J L}-\mathrm{O} 4$ & $\mathbf{J} \mathbf{L}-\mathrm{O} 3$ & Trained rare & AC, DF, GI, JL \\
\hline
\end{tabular}

A-L represent different symptom cues, O1-O4 represent different disease outcomes. Cues in italics indicate perfect predictors of common outcomes, and cues in bold indicate perfect predictors of rare outcomes. "Base rate" refers to the relative number of presentations of each trial type during training.

example, $\mathrm{O} 1$ was paired with the common $\mathrm{AB}$ and the rare $\mathrm{DF}$, while $\mathrm{O} 2$ was paired with the rare $\mathrm{AC}$ and the common DE. In this way, the local base-rate difference within each overlapping set (e.g., AB and AC) is maintained, but there is no global difference in the frequencies of each outcome. Importantly, at any point in the experiment, all outcomes will have been experienced at equal rates, eliminating the potential influence of early-learning biases. If outcome novelty is important for the effect, we would expect the preference for the rare outcome on conflicting trials to be much stronger in the standard than in the balanced group.

\section{Method}

Participants Eighty-three students participated in return for partial course credit or monetary compensation. Four participants were excluded for failing to meet the training criterion. Seventy-nine participants ( 56 female; mean age $=21.28$ years, $S D=4.09)$ remained, randomly allocated to the standard $(n=39)$ and balanced $(n=40)$ conditions.

Apparatus and stimuli The apparatus and symptom cues were identical to those in Experiment 1. The four disease outcomes included buragamo, coralgia, midosis, and terrigitis.

Design The design is shown in Table 4. The only difference between the standard and balanced groups was the overall frequency of each outcome during training. The standard group was identical to the shared-outcome group in Experiment 1. In the balanced group, each of the four outcomes was paired with one common and one rare cue compound, such that all outcomes were experienced at equal rates throughout the experiment.
Procedure The training and transfer phases were completed in an identical manner to those in Experiment 1.

\section{Results}

The transfer results for critical trials are shown in Fig. 3, and those for all items are in Table 5. A significant rare bias was apparent in the standard group, $t(38)=5.59, p<.001, d=$ $0.89, \mathrm{BF}_{10}=11.7 \times 10^{4}$. Although rare choice on conflicting trials was numerically above chance in the balanced group, this did not reach significance, $t(39)=1.27, p=.211, d=0.20$, $\mathrm{BF}_{10}=0.98$. Importantly, the effect was significantly stronger in the standard than in the balanced group, $t(73.12)=2.44, p=$ $.017, d=0.55, \mathrm{BF}_{10}=10.74$. We observed a commonoutcome bias on imperfect trials in both the standard, $t(38)=$ $-9.37, p<.001, d=-1.50$, and the balanced, $t(39)=-7.56, p<$ $.001, d=-1.19$, groups. On combined trials, choice did not differ from chance in the standard group, $t(38)=1.57, p=$ $125, d=0.25$, but there was a significant common-outcome bias in the balanced group, $t(39)=-4.01, p<.001, d=-0.63$, and this group difference was significant, $t(77)=3.80, p<$ $.001, d=0.85$.

Again, novel trials showed a common-outcome bias in both the standard, $t(38)=-6.15, p<.001, d=-0.98$, and balanced, $t(39)=-3.83, p<.001, d=-0.61$, groups. As in Experiment 1 , the proportion of rare responses on novel trials was significantly higher than on imperfect trials, $F(1,77)=15.32, p<.001, \eta^{2}=$ .06 , but significantly lower than on conflicting trials, $F(1,77)$ $=55.03, p<.001, \eta^{2}=.28$. This difference between novel and conflicting trials was significantly greater in the standard than in the balanced group, $F(1,77)=5.65, p=.02, \eta^{2}=.03$.

\section{Discussion}

When all outcomes were experienced at equal rates in the balanced condition, such that no one outcome was more novel 


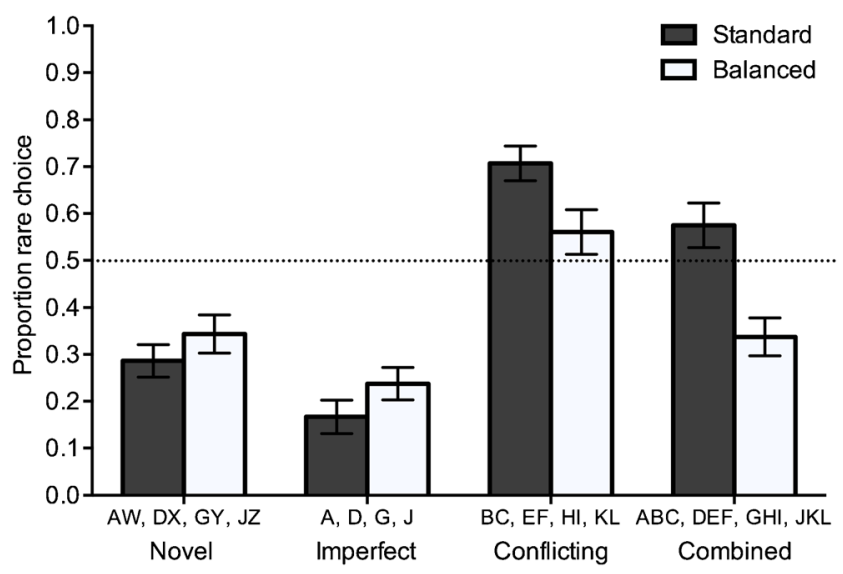

Fig. 3 Mean proportion of relevant rare-outcome choice $( \pm S E M)$ for the critical transfer items in Experiment 2

than another, the inverse base-rate effect was considerably weakened relative to the standard condition. This result indicates that global outcome frequency differences are important for the effect, because outcome novelty influenced rare choices on conflicting trials.

Still, the overall pattern of results is somewhat problematic for a general novelty-matching explanation. Responses to novel cues again favored the common outcome in both conditions. Medin and Edelson (1988) ruled out novelty selection in its simplest form, because the combined cues (ABC trials) showed a common-outcome bias. Nevertheless, in Experiment 2, we observed significant differences in responding to the combined trials between groups, with the standard group making fewer common choices than the balanced group. This result may suggest that a novelty bias contributes to choices on the combined transfer trials to some extent.

Taken together, the results of Experiment 2 may instead suggest that a preference for a novel outcome is driven by "prediction conflict" in the transfer trial, and resulting uncertainty, rather than novelty per se. The conflicting trials consist of two cues that are both equally predictive of their respective outcomes, and therefore the correct response is unclear. The imperfect predictor and the novel compound contain a cue that was paired with both a common and a rare outcome, and thus some conflict might also occur on these trials. However, this conflict may be substantially weaker, since the imperfect predictor was not consistently paired with a single outcome during training. The combined transfer trials also contain conflicting information, yet often elicit common-outcome choices (albeit not as consistently as the biases observed for conflicting and imperfect trials). In this case, the addition of the rare predictor may tip the weight of evidence in favor of the common outcome in some cases, and may fail to do so in others. This explanation suggests that strong conflict between equivalent predictors of the cues presented on test and a global outcome frequency difference may be jointly sufficient to produce the effect. However, several studies have failed to find an effect under these conditions, when two predictors of different outcomes (one common, one rare) that were not trained with a shared cue were combined at test (Kruschke, 2001a; Medin \& Edelson, 1988; Wills et al., 2014). Their results suggest that cue overlap in the training trials, producing at least some conflict in outcome predictions

Table 5 Outcome choice proportions and mean confidence ratings for all transfer trials in Experiment 2

\begin{tabular}{|c|c|c|c|c|c|c|c|}
\hline \multirow[b]{2}{*}{ Transfer Trial } & \multirow[b]{2}{*}{ Group } & \multicolumn{5}{|c|}{ Choice Proportion } & \multirow[b]{2}{*}{ Confidence } \\
\hline & & Common & Rare & Other Common & Other Rare & Other & \\
\hline Imperfect & Standard & .788 & .154 & .045 & .013 & & 59.45 \\
\hline $\mathrm{A}, \mathrm{D}, \mathrm{G}, \mathrm{J}$ & Balanced & .725 & .225 & & & .050 & 56.27 \\
\hline Conflicting & Standard & .288 & .692 & 0 & .019 & & 68.75 \\
\hline BC, EF, HI, KL & Balanced & .400 & .538 & & & .063 & 60.83 \\
\hline Combined & Standard & .423 & .571 & 0 & .006 & & 70.84 \\
\hline ABC, DEF, GHI, JKL & Balanced & .644 & .338 & & & .019 & 66.54 \\
\hline Novel & Standard & .654 & .269 & .032 & .045 & & 55.29 \\
\hline AW, DX, GY, JZ & Balanced & .581 & .300 & & & .119 & 47.86 \\
\hline Perfect common & Standard & .942 & .013 & .032 & .013 & & 71.36 \\
\hline $\mathrm{B}, \mathrm{E}, \mathrm{H}, \mathrm{K}$ & Balanced & .963 & .013 & & & .025 & 71.13 \\
\hline Perfect Rare & Standard & .038 & .936 & 0 & .026 & & 80.56 \\
\hline $\mathrm{C}, \mathrm{F}, \mathrm{I}, \mathrm{L}$ & Balanced & .063 & .838 & & & .100 & 68.67 \\
\hline Trained common & Standard & 1 & 0 & 0 & 0 & & 92.86 \\
\hline $\mathrm{AB}, \mathrm{DE}, \mathrm{GH}, \mathrm{JK}$ & Balanced & .988 & .013 & & & 0 & 91.42 \\
\hline Trained rare & Standard & .051 & .929 & 0 & .019 & & 88.40 \\
\hline AC, DF, GI, JL & Balanced & .081 & .900 & & & .019 & 82.87 \\
\hline
\end{tabular}


during training, may also be critical for the inverse base-rate effect.

\section{Experiment 3}

Experiment 3 tested whether a global outcome frequency difference was sufficient to produce a rare bias for conflicting cues, even without critical-trial frequency differences, provided that a shared cue was still used during training. That is, even if the two predictors that comprise the conflicting test trial were themselves presented with equal frequencies, an effect might still be possible based on the overall novelty of the outcome, provided that prediction conflict is present during both training (because of the shared cue A) and test (because of the combination of two perfect predictors B and C).

The standard condition from Experiment 2 provided a suitable control, since the difference in outcome frequencies occurrs in conjunction with the difference in trial frequencies. This was compared to an "equal-trial" group that equated trial frequency while maintaining outcome frequency differences. In this group, $\mathrm{AB}-\mathrm{O} 1$ and $\mathrm{AC}-\mathrm{O} 2$ trials were presented at an equal, 1:1 base rate. The frequency of one outcome was increased by including high-frequency filler cue compounds paired with that outcome-for example, ST-O1. Because $\mathrm{AB}$ and $\mathrm{AC}$ were both presented at equal frequencies, cuecompetition theories would predict no bias on conflicting $\mathrm{BC}$ trials, since each contingency would be learned at an equal rate. However, B was paired with an outcome that was otherwise experienced at a greater frequency than the outcome paired with $\mathrm{C}$. If the inverse base-rate effect is simply the result of matching conflicting cues to rare outcomes, the experience of a global outcome frequency difference should produce an effect in the equal-trial group.

\section{Method}

Participants Sixty-one students participated in return for partial course credit. Three participants were excluded for failing to meet the training criterion, leaving 58 participants (44 female; mean age $=19.33$ years, $S D=2.53$ ), randomly allocated to each group $(n=29)$.

Apparatus and stimuli The apparatus was identical to those in previous experiments. The 18 symptoms cues were randomly allocated from the following 20 symptoms: sneezing, dizziness, headache, fever, rash, nausea, earache, coughing, hair loss, fatigue, twitching, blurred vision, wheezing, swelling, back pain, fainting, stomach cramps, slurred speech, puffy eyes, and bloody nose. The disease outcomes were identical to those in Experiment 2.
Design The design is presented in Table 6. The standard group was compared to an equal-trial group in which all critical $\mathrm{AB} /$ $\mathrm{AC}$ trials were presented at a 1:1 base rate, while the frequency of the common outcome was increased through pairings with filler compounds. To maintain the number of trials and the 3:1 outcome base rate in the equal-trial group, each of the critical contingencies was presented twice per block, and each of the filler compounds was presented eight times per block. Thus, in both groups each common outcome was presented 12 times per block, and each rare outcome was presented four times per block. The filler compounds were also included in the transfer phase for the equal-trial group.

Procedure The task proceeded in the same manner as in previous experiments.

\section{Results}

In the following analyses, $\mathrm{AB}, \mathrm{DE}, \mathrm{GH}$, and $\mathrm{JK}$ will still be referred to as the common trial types and $\mathrm{AC}, \mathrm{DF}, \mathrm{GI}$, and $\mathrm{JL}$ as the rare trial types, according to the frequency of the outcome with which they were paired. The proportions of rare choice for the critical transfer items are shown in Fig. 4, and those for all items are in Table 7 . We found a significant rare bias in the standard group, $t(28)=3.81, p=.001, d=0.71$, $\mathrm{BF}_{10}=24.5 \times 10^{3}$, but not in the equal-trial group, $t(28)=$ $0.82, p=.417, d=0.15, \mathrm{BF}_{01}=4.40$, and this group difference was significant, $t(56)=2.30, p=.025, d=0.60, \mathrm{BF}_{10}=42.11$.

We also observed significant group differences on the imperfect, $t(56)=-4.16, p<.001, d=-1.09$, and novel, $t(56)=-$ $2.40, p=.02, d=-0.63$, trials. In the standard group, there was a common bias for imperfect trials, $t(28)=-7.28, p=<.001, d$ $=-1.35$, and novel trials, $t(28)=-4.58, p=.001, d=-0.85$. Choice on the combined trials did not differ from chance, $t(28)$ $=-0.26, p=.796, d=-0.05$. In the equal-trial group, outcome choice did not differ from chance for any of the critical transfer trials, highest $t(28)=-1.21, p=.238, d=-0.22$, for novel trials.

Across both groups, rare choice on novel trials did not differ significantly from the imperfect trials, $F<1$, but was significantly lower than on conflicting trials, $F(1,56)=23.50$, $p<.001, \eta^{2}=.20$. A significant interaction with group also emerged, $F(1,56)=9.22, p=.004, \eta^{2}=.08$, indicating a greater difference in the standard than in the equal-trial group.

\section{Discussion}

In Experiment 3, we aimed to determine whether global outcome frequency differences and conflicting cue-outcome combinations are jointly sufficient to produce an inverse base-rate effect, despite equal frequencies of the relevant predictive-cue trials. When $\mathrm{AB}-\mathrm{O} 1$ and $\mathrm{AC}-\mathrm{O} 2$ were trained with equal base rates and the frequency of $\mathrm{O} 1$ was 
Table 6 Design of Experiment 3

\begin{tabular}{|c|c|c|c|c|c|}
\hline \multicolumn{4}{|c|}{ Training Phase } & \multicolumn{2}{|l|}{ Transfer Phase } \\
\hline \multicolumn{2}{|l|}{ Standard } & \multicolumn{2}{|l|}{ Equal-Trial } & \multirow[b]{2}{*}{ Type } & \multirow[b]{2}{*}{ Trials } \\
\hline Base Rate & Trials & Base Rate & Trials & & \\
\hline 3 & $\mathrm{~A} B-\mathrm{O} 1$ & 1 & $\mathrm{~A} B-\mathrm{O} 1$ & Imperfect predictors & $\mathrm{A}, \mathrm{D}, \mathrm{G}, \mathrm{J}$ \\
\hline 1 & $\mathrm{AC}-\mathrm{O} 2$ & 1 & $\mathrm{AC}-\mathrm{O} 2$ & Conflicting transfer & $B \mathbf{C}, E \mathbf{F}, H \mathbf{I}, K \mathbf{L}$ \\
\hline 3 & $\mathrm{D} E-\mathrm{O} 1$ & 1 & $\mathrm{D} E-\mathrm{O} 1$ & Combined transfer & $\mathrm{A} B \mathbf{C}, \mathrm{D} E \mathbf{F}, \mathrm{G} H \mathbf{I}, \mathrm{J} K \mathbf{L}$ \\
\hline 1 & $\mathrm{DF}-\mathrm{O} 2$ & 1 & $\mathrm{DF}-\mathrm{O} 2$ & Novel transfer & AW, DX, GY, JZ \\
\hline 3 & $\mathrm{G} H-\mathrm{O} 3$ & 1 & $\mathrm{G} H-\mathrm{O} 3$ & Perfect common & $B, E, H, K$ \\
\hline 1 & GI-O4 & 1 & GI-O4 & Perfect rare & $\mathbf{C}, \mathbf{F}, \mathbf{I}, \mathbf{L}$ \\
\hline 3 & $\mathrm{~J} K-\mathrm{O} 3$ & 1 & $\mathrm{~J} K-\mathrm{O} 3$ & Trained common & $\mathrm{A} B, \mathrm{D} E, \mathrm{G} H, \mathrm{~J} K$ \\
\hline \multirow[t]{3}{*}{1} & $\mathrm{JL}-\mathrm{O} 4$ & 1 & $\mathrm{JL}-\mathrm{O} 4$ & Trained rare & AC, DF, GI, JL \\
\hline & & 4 & $\mathrm{ST}-\mathrm{O} 1$ & Filler cues & ST, UV \\
\hline & & 4 & $\mathrm{UV}-\mathrm{O} 3$ & & \\
\hline
\end{tabular}

A-V represent different symptom cues, O1-O4 represent different disease outcomes. Cues in italics indicate perfect predictors of common outcomes, and cues in bold indicate perfect predictors of rare outcomes. Filler-cue transfer trials were presented in the equal-trial condition only. "Base rate" refers to the relative number of presentations of each trial type during training.

independently increased through pairings with highfrequency filler trials, the effect was substantially reduced relative to the standard condition. Responding to all other critical transfer trials also did not differ from chance in this condition. This result indicates that although global outcome frequency differences are important, the effect is not simply the result of matching conflicting cues with a novel outcome. Taken together, Experiments 2 and 3 suggest that a combination of differences in both relevant-trial frequency and global outcome frequency are important for producing the inverse base-rate effect.

The results are consistent with Johansen et al.'s (2007) conclusion that asymmetric representations are necessary for the effect. That is, $\mathrm{O} 1$ is predicted by both $\mathrm{A}$ and $\mathrm{B}$, whereas $\mathrm{O} 2$ is primarily predicted by $\mathrm{C}$. Differences in trial frequencies serve to establish this asymmetry, as $\mathrm{AB}-\mathrm{O} 1$ is learned faster

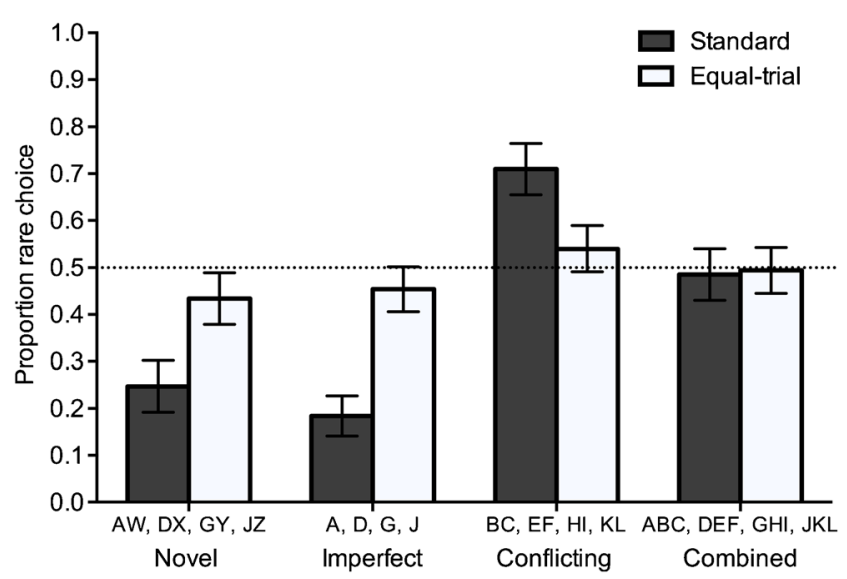

Fig. 4 Mean proportion of relevant rare-outcome choice $( \pm S E M)$ for the critical transfer items in Experiment 3 than $\mathrm{AC}-\mathrm{O} 2$. In the equal-trial condition, asymmetric representation is lost, since the imperfect predictor would become equally well associated with both outcomes. Indeed, the typical common-outcome bias for the imperfect predictor was not observed in this condition. Novel-trial responses, which could be guided by imperfect cue associations, also showed no bias.

Interestingly, participants were slightly slower to learn $\mathrm{AB}$ trials than $\mathrm{AC}$ trials in the equal-trial group, even though they were both presented at equal rates throughout training (see the supplementary materials). This finding may indicate a role of context learning in outcome frequency manipulations. It is possible that the context became associated with the common outcome, which might then have blocked learning for the relationship between $\mathrm{AB}$ and the common outcome. We will consider the implications of context learning further in the General Discussion. However, for this experiment, the difference in acquisition is unlikely to have affected performance at test, because accuracy for $\mathrm{AB}$ and $\mathrm{AC}$ trials was equivalent by the end of training.

\section{General discussion}

In the present study, we manipulated the relative frequencies of outcomes and cues to investigate their importance for the inverse base-rate effect. Perhaps the most striking finding was that a combination of outcome frequency differences and specific trial frequency differences is important for the effect, which was substantially weakened when either of these biases was independently removed in Experiments 2 and 3. We will first summarize the influences of these manipulations on the 
Table 7 Outcome choice proportions and mean confidence ratings for all transfer trials in Experiment 3

\begin{tabular}{|c|c|c|c|c|c|c|}
\hline \multirow[b]{2}{*}{ Transfer Trial } & \multirow[b]{2}{*}{ Group } & \multicolumn{4}{|c|}{ Choice Proportion } & \multirow[b]{2}{*}{ Confidence } \\
\hline & & Common & Rare & Other Common & Other Rare & \\
\hline Imperfect & Standard & .784 & .164 & .026 & .026 & 62.25 \\
\hline $\mathrm{A}, \mathrm{D}, \mathrm{G}, \mathrm{J}$ & Equal-trial & .483 & .440 & .043 & .034 & 61.94 \\
\hline Conflicting & Standard & .250 & .672 & .069 & .009 & 67.86 \\
\hline BC, EF, HI, KL & Equal-trial & .431 & .491 & .034 & .043 & 68.86 \\
\hline Combined & Standard & .483 & .483 & 0 & .034 & 71.90 \\
\hline ABC, DEF, GHI, JKL & Equal-trial & .474 & .466 & .043 & .017 & 72.38 \\
\hline Novel & Standard & .690 & .198 & .060 & .052 & 50.79 \\
\hline AW, DX, GY, JZ & Equal-trial & .483 & .388 & .078 & .052 & 54.09 \\
\hline Perfect common & Standard & .897 & .043 & .034 & .026 & 71.80 \\
\hline $\mathrm{B}, \mathrm{E}, \mathrm{H}, \mathrm{K}$ & Equal-trial & .784 & .095 & .060 & .060 & 71.79 \\
\hline Perfect rare & Standard & .112 & .828 & .017 & .043 & 75.13 \\
\hline $\mathrm{C}, \mathrm{F}, \mathrm{I}, \mathrm{L}$ & Equal-trial & .095 & .802 & .060 & .043 & 73.87 \\
\hline Trained common & Standard & .759 & .198 & .043 & 0 & 82.31 \\
\hline $\mathrm{AB}, \mathrm{DE}, \mathrm{GH}, \mathrm{JK}$ & Equal-trial & .767 & .207 & 0 & .026 & 83.37 \\
\hline Trained rare & Standard & .112 & .828 & .017 & .043 & 85.30 \\
\hline AC, DF, GI, JL & Equal-trial & .095 & .802 & .060 & .043 & 85.21 \\
\hline
\end{tabular}

effect, before discussing the theoretical implications of these findings.

The similarity of transfer trials to the trained contingencies does not appear to influence the likelihood of choosing the rare outcome in a straightforward way. All three experiments included novel transfer trials that were at least as dissimilar to the trained trials as were the conflicting transfer trials. In all but one condition (in which no bias was shown), the relevant common outcome was more likely to be chosen. Experiment 2 demonstrated that relative outcome frequency differences are important for the effect: Preference for the rare outcome was reduced when each outcome had been experienced at an equal rate, even when trial frequency differences of the relevant cue compounds were maintained. Relative trial frequency differences nevertheless also appear to be important: The presence of conflicting predictors and a global outcome frequency difference were not sufficient to produce the effect when those predictors were experienced at equal frequencies during training in Experiment 3.

\section{Inferential processes}

In these experiments, we aimed to test reasoning processes that could plausibly contribute to the inverse base-rate effect. Although eliminative inference does not capture all characteristics of the effect, previously demonstrated novel-cue effects (Johansen et al., 2007; Juslin et al., 2001) suggest that some form of outcome elimination or novelty matching might still be involved on conflicting trials. If this were the case, then rare outcome choice should increase according to the novelty of transfer trials, as compared to learned instances. Overall, our results are inconsistent with these explanations. In all three experiments, the novel transfer cues were consistently biased toward the common outcome, with the exception of the equaltrial condition in Experiment 3, which showed no bias. Moreover, this common-response bias only occurred in conditions in which an inverse base-rate effect was also observed, and not in the absence of an effect (Exp. 3). This is an important observation, because rare biases for single novel cues have only been demonstrated in conjunction with a nonsignificant inverse base-rate effect (Johansen et al., 2007; Juslin et al., 2001). Nevertheless, rare responses were greater to novel than to imperfect trials in Experiments 1 and 2, which may suggest at least some influence of novelty on rare responding.

Additionally, the influence of global outcome frequency differences indicates that outcome novelty is important for the effect, and might suggest the contribution of a novelty selection process. When presented with conflicting cues, participants were more likely to predict the relevant rare outcome if that outcome was experienced less often over the entire course of the experiment. When participants experienced each outcome at an equal rate, such that neither outcome could provide a "novel" response, the effect was considerably weakened. The combination of this result with common biases on novel trials could suggest that novelty selection only occurs under conditions of uncertainty based on prediction conflict in transfer trials, as opposed to the novelty of transfer trials. Although some conflict may occur on other critical transfer trials, this may be weaker than the combination of two equivalent predictors of conflicting outcomes (see Exp. 2 
discussion). However, other results indicate that this explanation is also insufficient. Confidence ratings on conflicting trials were consistently high relative to both the imperfect and novel trials (see the results tables and the supplementary materials), which shows that participants were not uncertain about their choices on conflicting trials. Moreover, the experience of a conflicting combination of predictive cues and outcome frequency differences in the equal-trial group of Experiment 3 failed to elicit the effect. Here, no bias was observed when the relevant cues had been experienced at equal rates during training. Experiment 3 therefore demonstrates that relative trial frequency differences during training are also critical for the effect, in a way that is not consistent with adopting novelty selection as a general strategy at test.

\section{Cue competition and the EXIT model}

Although the present experiments were not designed to test the predictions of EXIT, we will also consider how the results fit within this theoretical framework (Kruschke, 1996, 2001a). Learning about the imperfect cue is crucial for prediction error accounts of the inverse base-rate effect. According to EXIT, the imperfect predictor is more strongly associated with the common outcome, which then elicits a shift in attention toward the perfect predictor on rare trials. Assuming that the imperfect predictor would control responding, this account can readily explain the common-outcome bias for novel transfer trials. The necessity of relative trial frequency differences observed in Experiment 3 is also clearly consistent with EXIT, since the imperfect predictor would become equally associated with both outcomes in the equal-trial condition.

However, EXIT should also predict an effect in the balanced group of Experiment 2, since the difference in relative trial frequencies during training would allow the imperfect predictor to become strongly associated with the relevant common outcome, regardless of the global outcome frequency differences. Outcome frequency effects may be consistent with attention-based explanations if we assume a strong role of context learning. EXIT permits learning about base rates in a cue-independent way by using "bias" units, which essentially serve as a background context that can become associated with the outcomes. In the standard condition, the context could become strongly associated with the common outcome, which might further encourage attention shifts toward the predictive cue on rare trials. Yet in the balanced condition, in which each outcome was experienced equally, the context does not provide a greater expectation of either outcome, such that prediction error and subsequent attention shifts on rare trials may be reduced, consequently weakening the inverse base-rate effect.

It is difficult to reconcile this explanation with the results of Experiment 3. Learning for $\mathrm{AB}-\mathrm{O} 1$ appeared to be somewhat blocked during training, which suggests that context conditioning does occur. However, a strong association between the context and the common outcome (due to the high-frequency filler cues) should then facilitate rare choice, which did not occur. It is possible that both context and imperfect-cue associations are necessary to drive an attention shift strong enough to influence responding at test, whereas each alone is insufficient. Clearly determining the role of context learning nevertheless remains a challenge for future research. In summary, an explanation based on competitive associative learning and attention biases provides a reasonable but still incomplete account of the present results.

\section{Sequential guessing strategies}

Participants might employ a different kind of guessing strategy on ambiguous test trials. When participants are uncertain about a response, they might simply guess an outcome they have not recently chosen. Due to the nature of the task, participants tend to make fewer rare-outcome than commonoutcome responses. Thus, on conflicting trials, participants might select the rare outcome simply because they have not chosen it recently. If this is the case, participants should be more likely to show a rare bias on conflicting trials when they have made a greater number of common outcome choices on the preceding trials. To assess this possibility, we determined the probability of choosing the rare outcome on conflicting trials as a function of the number of trials since last selecting that outcome, collapsed across the standard group of each experiment (see Fig. 5). A simple linear regression revealed that the number of trials did not significantly predict the probability of selecting the relevant rare outcome, $\beta=-.21, F<1$. Furthermore, the probability of choosing the rare outcome on conflicting trials was consistently above $50 \%$, regardless of the number of trials since choosing that outcome, suggesting

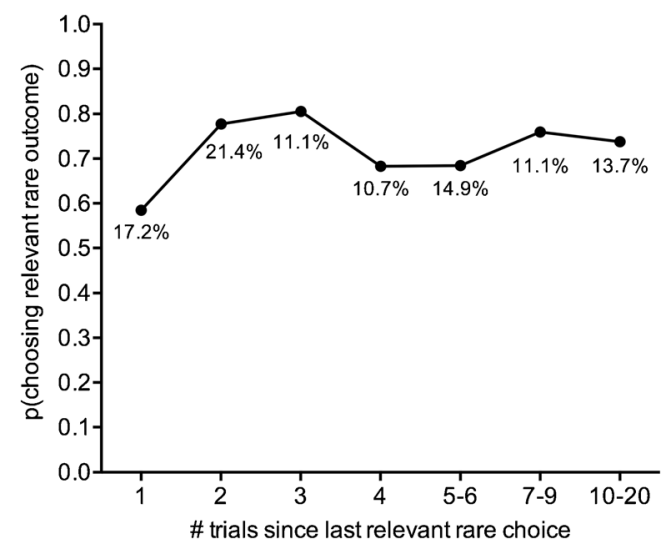

Fig. 5 Probabilities of choosing the relevant rare outcome on conflicting trials (i.e., showing an inverse base-rate effect) as a function of the number of trials since previously choosing that outcome, for participants in the standard condition of each experiment (including the shared-outcome condition of Exp. 1). The data labels indicate the percentages of cases 
that the effect is not driven by a guessing strategy influenced by sequential choice factors.

\section{The development of inferences (and associations) during learning}

Given that the inverse base-rate effect only occurs following a specific set of training conditions sensitive to global outcome frequency, trial frequency, and the presence of a shared cue, we argue that any inferential decision bias that is generated de novo at test will struggle to account for the data. Nevertheless, it is still possible that inferential processes form during training, which then influence the outcome choice at test. If this were the case, it would not be surprising that training parameters affect the way in which those inferences are formed and applied. For instance, during training, participants might discover that cue $\mathrm{C}$ on $\mathrm{AC}$ trials means that $\mathrm{O} 1$ will not occur, and this inference might then generalize to $\mathrm{BC}$ trials at test, resulting in greater $\mathrm{O} 2$ responses. A simple inhibitory association also captures this relationship, and thus it may seem unnecessary to describe it specifically as an inference, or to assume that it only occurs under very specific conditions. However, in similar paradigms, learning inhibitory relationships has proved to be surprisingly sensitive to training conditions (Karazinov \& Boakes, 2007; Lee \& Livesey, 2012). Thus, it might take the combination of trial frequency differences and global outcome frequency differences for a strong inhibitory relationship between $\mathrm{C}-\mathrm{O} 1$ to be learned (either inferentially or otherwise). An inference or an inhibitory association of this nature might only arise if early AC trials produce a strong erroneous expectation of the common outcome, provided at least in part by the imperfect predictor A. This would be consistent with the necessity of trial frequency differences, as well as asymmetric representation and a shared cue during training (Johansen et al., 2007; Kruschke, 2001a; Wills et al., 2014). Furthermore, it could also explain why the effect occurs under conditions of prediction conflict, and not of trial novelty.

Given the mixed findings in the literature (e.g., Lamberts \& Kent, 2007; Winman et al., 2005) and the present results, it seems reasonable to assume that both higher-order inferences and associative attention processes are involved to some degree in generating the effect. Similar possibilities have been raised in relation to other learning phenomena that have been explained with recourse to attention biases. For instance, the learned-predictiveness effect displays some properties characteristic of intentional cognitive control, but at least some component appears to be the consequence of an automatic expression of prior learning (Don \& Livesey, 2015; Shone, Harris, \& Livesey, 2015). There is no reason to assume that the inverse base-rate effect is any different, in this regard. Thus, we suggest that the effect is not well characterized as a selection strategy at test, but rather as a consequence of the specific conditions under which the relationships between cues and outcomes are learned during training, regardless of whether that learning is inferential or otherwise.

\section{References}

Brainard, D. H. (1997). The psychophysics toolbox. Spatial Vision, 10, 433-436. doi:10.1163/156856897X00357

Don, H. J., Goldwater, M. B., Otto, A. R., \& Livesey, E. J. (2016). Rule abstraction, model-based choice, and cognitive reflection. Psychonomic Bulletin and Review, 23, 1615-1623. doi:10.3758/s13423-016-1012-y

Don, H. J., \& Livesey, E. J. (2015). Resistance to instructed reversal of the learned predictiveness effect. Quarterly Journal of Experimental Psychology, 68, 1327-1347.

Johansen, M. K., Fouquet, N., \& Shanks, D. R. (2007). Paradoxical effects of base rates and representation in category learning. Memory \& Cognition, 35, 1365-1379. doi:10.3758/BF03193608

Johansen, M. K., Fouquet, N., \& Shanks, D. R. (2010). Featural selective attention, exemplar representation, and the inverse base-rate effect. Psychonomic Bulletin \& Review, 17, 637643. doi:10.3758/PBR.17.5.637

Juslin, P., Wennerholm, P., \& Winman, A. (2001). High-level reasoning and base-rate use: Do we need cue-competition to explain the inverse baserate effect? Journal of Experimental Psychology: Learning, Memory, and Cognition, 27, 849-871. doi:10.1037/10278-7393.27.3.849

Kalish, M. L. (2001). An inverse base rate effect with continuously valued stimuli. Memory \& Cognition, 29(4), 587-597.

Karazinov, D. M., \& Boakes, R. A. (2004). Learning about cues that prevent an outcome: Conditioned inhibition and differential inhibition in human predictive learning. Quarterly Journal of Experimental Psychology, 57B, 153-178.

Karazinov, D. M., \& Boakes, R. A. (2007). Second-order conditioning in human predictive judgements when there is little time to think. Quarterly Journal of Experimental Psychology, 60, 448-460. doi:10.1080/17470210601002488

Kruschke, J. K. (1996). Base rates in category learning. Journal of Experimental Psychology: Learning, Memory, and Cognition, 22, 3-26. doi:10.1037/0278-7393.22.1.3

Kruschke, J. K. (2001a). The inverse base-rate effect is not explained by eliminative inference. Journal of Experimental Psychology: Learning, Memory, and Cognition, 27, 1385-1400. doi:10.1037/102787393.27.6.1385

Kruschke, J. K. (2001b). Toward a unified model of attention in associative learning. Journal of Mathematical Psychology, 45, 812-863. doi:10.1006/jmps.2000.1354

Kruschke, J. K. (2003). Attentional theory is a viable explanation of the inverse base rate effect: A reply to Winman, Wennerholm, and Juslin (2003). Journal of Experimental Psychology: Learning, Memory, and Cognition, 29, 1396-1400. doi:10.1037/0278-7393.29.6.1396

Kruschke, J. K. (2009). Highlighting: A canonical experiment. In B. H. Ross (Ed.), The psychology of learning and motivation (Vol. 51, pp. 153-185). San Diego, CA: Academic Press. doi:10.1016/S00797421(09)51005-5

Kruschke, J. K., Kappenman, E. S., \& Hetrick, W. P. (2005). Eye gaze and individual differences consistent with learned attention in associative blocking and highlighting. Journal of Experimental Psychology: Learning, Memory, and Cognition, 31, 830-845. doi:10.1037/0278-7393.31.5.830 
Lamberts, K., \& Kent, C. (2007). No evidence for rule-based processing in the inverse base-rate effect. Memory \& Cognition, 35, 20972105. doi:10.3758/BF03192941

Lee, J. C., \& Livesey, E. J. (2012). Second-order conditioning and conditioned inhibition: Influences of speed versus accuracy on human causal learning. PLoS ONE, 7, e49899. doi:10.1371/journal. pone.0049899

Le Pelley, M. E., Mitchell, C. J., Beesley, T., George, D. N., \& Wills, A. J. (2016). Attention and associative learning in humans: An integrative review. Psychological Bulletin, 142, 1111-1140. doi:10.1037/bul0000064

Lotz, A., \& Lachnit, H. (2009). Extinction of conditioned inhibition: Effects of different outcome continua. Learning \& Behavior, 37, 85-94. doi:10.3758/LB.37.1.85

McLaren, I. P., Forrest, C. L. D., McLaren, R. P., Jones, F. W., Aitken, M. R. F., \& Mackintosh, N. J. (2014). Associations and propositions: The case for a dual-process account of learning in humans. Neurobiology of Learning and Memory, 108, 185-195.

Medin, D. L., \& Bettger, J. G. (1991). Sensitivity to changes in base-rate information. American Journal of Psychology, 104, 311-332.

Medin, D. L., \& Edelson, S. M. (1988). Problem structure and the use of base-rate information from experience. Journal of Experimental Psychology: General, 117, 68-85. doi:10.1037/0096-3445.117.1.68

Mitchell, C. J., De Houwer, J., \& Lovibond, P. F. (2009). The propositional nature of human associative learning. Behavioural and Brain Sciences, 32, 183-198. doi:10.1017/S0140525X09000855. disc. 198-246.
Pelli, D. G. (1997). The Video Toolbox software for visual psychophysics: Transforming numbers into movies. Spatial Vision, 10, 437 442. doi: $10.1163 / 156856897 X 00366$

Shone, L. T., Harris, I. M., \& Livesey, E. J. (2015). Automaticity and cognitive control in the learned predictiveness effect. Journal of Experimental Psychology: Animal Learning and Cognition, 41, $18-31$.

Wills, A. J., Barrasin, T. J., \& McLaren, I. P. L. (2011). Working memory capacity and generalization in predictive learning. In L. Carlson, C. Hölscher, \& T. F. Shipley (Eds.), Expanding the space of cognitive science: Proceedings of the 33rd Annual Conference of the Cognitive Science Society (pp. 3205-3210). Austin, TX: Cognitive Science Society.

Wills, A. J., Lavric, A., Hemmings, Y., \& Surrey, E. (2014). Attention, predictive learning, and the inverse base-rate effect: Evidence from event-related potentials. NeuroImage, 87, 61-71. doi:10.1016/j. neuroimage.2013.10.060

Winman, A., Wennerholm, P., Juslin, P., \& Shanks, D. R. (2005). Evidence for rule-based processes in the inverse base-rate effect. Quarterly Journal of Experimental Psychology, 58A, 789-815. doi:10.1080/02724980443000331

Winman, A., Wennerholm, P., \& Juslin, P. (2003). Can attentional theory explain the inverse base rate effect? Comment on Kruschke (2001). Journal of Experimental Psychology: Learning, Memory, and Cognition, 29, 1390-1395. doi:10.1037/0278-7393.29.6.1390 